\title{
Combining macula clinical signs and patient characteristics for age-related macular degeneration diagnosis: a machine learning approach
}

\author{
Paolo Fraccaro ${ }^{1,2,6,7}$, Massimo Nicolo ${ }^{4^{*}}$, Monica Bonetto ${ }^{3,5}$, Mauro Giacomini ${ }^{3,5}$, Peter Weller ${ }^{1}$, Carlo Enrico Traverso ${ }^{4}$, \\ Mattia Prosperi ${ }^{2,7}$ and Dympna O'Sullivan ${ }^{1}$
}

\begin{abstract}
Background: To investigate machine learning methods, ranging from simpler interpretable techniques to complex (non-linear) "black-box" approaches, for automated diagnosis of Age-related Macular Degeneration (AMD).

Methods: Data from healthy subjects and patients diagnosed with AMD or other retinal diseases were collected during routine visits via an Electronic Health Record (EHR) system. Patients' attributes included demographics and, for each eye, presence/absence of major AMD-related clinical signs (soft drusen, retinal pigment epitelium, defects/ pigment mottling, depigmentation area, subretinal haemorrhage, subretinal fluid, macula thickness, macular scar, subretinal fibrosis). Interpretable techniques known as white box methods including logistic regression and decision trees as well as less interpreitable techniques known as black box methods, such as support vector machines (SVM), random forests and AdaBoost, were used to develop models (trained and validated on unseen data) to diagnose AMD. The gold standard was confirmed diagnosis of AMD by physicians. Sensitivity, specificity and area under the receiver operating characteristic (AUC) were used to assess performance.

Results: Study population included 487 patients (912 eyes). In terms of AUC, random forests, logistic regression and adaboost showed a mean performance of (0.92), followed by SVM and decision trees (0.90). All machine learning models identified soft drusen and age as the most discriminating variables in clinicians' decision pathways to diagnose AMD.

Conclusions: Both black-box and white box methods performed well in identifying diagnoses of AMD and their decision pathways. Machine learning models developed through the proposed approach, relying on clinical signs identified by retinal specialists, could be embedded into EHR to provide physicians with real time (interpretable) support.
\end{abstract}

Keywords: Age related macular degeneration, Machine learning, Automated diagnosis, Statistical learning, macula disease

\section{Background}

Age-related macular degeneration (AMD) is the leading cause of severe reduction in central visual acuity in adults aged 50 years and older in developed countries [1]. As the prevalence of AMD is steadily increasing due to increasing life expectancy [2], early diagnosis and treatment becomes essential in slowing down progression of AMD and subsequent vision loss [3]. Multimodal high-resolution imaging has had a substantial impact on diagnosis and treatment

\footnotetext{
* Correspondence: massimonicolo@gmail.com

${ }^{4}$ Di.N.O.G.Mi, University of Genoa, L.go P. Daneo 3, Genoa 16132, Italy

Full list of author information is available at the end of the article
}

of macular diseases [4]. Different imaging modalities can be used for AMD diagnosis [5]. In particular, optical coherence tomography (OCT) associated with color fundus image acquisition technology is a non-contact, non-invasive, high resolution technique which produces real-time images used to derive several features of the macula [6]. Such characteristics may allow OCT to become an effective screening instrument, employable in nonspecialized environments (such as pharmacies) and by nonspecialized personnel to perform automatic diagnosis of AMD

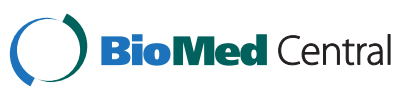

(c) 2015 Fraccaro et al.; licensee BioMed Central. This is an Open Access article distributed under the terms of the Creative Commons Attribution License (http://creativecommons.org/licenses/by/4.0), which permits unrestricted use, distribution, and reproduction in any medium, provided the original work is properly credited. The Creative Commons Public Domain Dedication waiver (http://creativecommons.org/publicdomain/zero/1.0/) applies to the data made available in this article, unless otherwise stated. 
without the intervention of a medical retinal specialist. However, to allow diagnosis by non-specialized personnel, OCT technology could be coupled with other clinical decision support functionalities [7] based on patient data which could enhance the potential of image analysis data. Currently, the majority of commercially available OCT technologies incorporate basic algorithms to automatically identify the presence of risk factors in macula images and diagnose AMD [8-10]. A recent review [5] showed how the majority of these algorithms mainly focus on automatic segmentation of soft drusen, previously identified as one of the most important signs for the diagnosis of AMD [11]. But relying on just one sign to diagnose AMD can be suboptimal since AMD is a complex pathology which involves different stages of progression and requires consideration of several clinical aspects [12]. Therefore image analysis should be used in conjunction with other clinical biomarkers to enhance diagnosis [5].

Machine learning techniques [13] have been applied successfully to identify, extract and analyze features in macula digital imaging [14-18]. In spite of potential higher accuracy in predicting disease diagnoses (as compared, for instance, to simple scoring rules on a small set of variables), many machine learning methods are usually regarded as non-transparent to the end user, and labeled as "black-boxes". Methods that do not allow the clinician to identify a clear decision pathway for the diagnosis are often regarded with skepticism in the clinical community [14].

This paper describes the application of a variety of more and less interpretable machine learning algorithms with the aims of: 1) reproducing physicians' diagnoses of AMD from patient data (demographics and clinical signs identified by retinal specialists through examination of medical images) and evaluating model performances on unseen data; 2) determining which are the diagnostic criteria followed by the physician (who may follow different routes to make a diagnosis); 3 ) identifying deviations or new rules that may emerge from the expected. Data were collected cross-sectionally from routine patient visits and stored in an Electronic Health Record, specifically designed for macular diseases management. The work reported in this paper is preliminary research into the potential of machine learning techniques to be used for AMD diagnostic support; from the perspective of using longitudinal data (i.e. symptoms/ markers before a diagnosis is made), these decision support algorithms could be embedded in Electronic Health Records to support physicians during everyday clinical practice, and could be further refined by incorporating features from digital image processing leading to a fullyautomated diagnostic tool suitable for non-specialized environments.

\section{Methods}

\section{Ethics statement}

The web Electronic Health Record system [19], data collection methods [20] and related observational studies were approved by an ethics committee (San Martino Hospital, Genoa, Italy) and patients signed informed consent for data storage and usage for clinical/research purposes.

\section{Study population and case selection}

Data on study participants, healthy subjects (those with normal macula) as well as patients with macular diseases (both referred to as "patients" in the text), were collected from March 2013 to January 2014 during routine clinical practice at the Medical Retina Center of the University Eye Clinic of Genoa (Italy).

The attributes used in the analysis included patient's age and gender, and for patient's left/right eye's:

- Primary diagnosis (AMD or other macular diseases);

- Relevant clinical signs [12] identified by clinicians during the visit as binary variables (positive if identified):

o Soft drusen;

o Retinal Pigment Epitelium (RPE) defects/pigment mottling;

o Depigmentation area(s);

o Subretinal haemorrhage;

o Subretinal fluid;

o Macula thickness;

o Macular scar;

o Subretinal fibrosis;

The macula of all eyes included in the study was evaluated by two different ophthalmologist (10 and 2 years of experience) using a spectral domain OCT machine (Topcon 3D OCT-2000, Topcon Medical Systems, Inc., Oakland, NJ, USA). Data records were stored per single eye.

The study population included a total of 487patients (912 eyes, with information on patients' two eyes not always available).

Primary diagnosis of AMD is the study outcome (dependent variable). Accordingly, each eye observation diagnosed as AMD was assigned to one class, while eyes diagnosed with other macular diseases were assigned to another class. The covariate set (input variables) included all the other attributes listed above and referred to the same eye.

There is evidence in support of the hypothesis of disease correlation between different eyes of the same patient [21]. A preliminary screening on our data confirmed this hypothesis. However, information on the fellow eye may not be available when diagnoses are performed during a visit (for example, it may be the first encounter). Therefore, we performed the analysis in this paper without 
taking into account the information about the presence of AMD in the fellow eye.

\section{Machine learning techniques}

The purpose of this section is not to provide a detailed explanation of machine learning methods, which is left to referenced works, but to give some introduction about the techniques which readers may be less familiar with [22]. All statistical analyses and graphs were done using the R software (www.r-project.org).

Logistic regression is used for predicting the outcome of a categorical dependent variable (i.e., a class label) based on one or more predictor variables. This "whitebox" technique is widely used in automatic medical diagnosis [22,23]. An embedded procedure within logistic regression, called the LogitBoost [24] (as implemented in RWeka R library [25]), was included to select the most relevant variables. No variable interactions were explored. For comparison purposes, a simple model based on a unique variable was employed, named "one-rule", selecting the most discriminative variable, based on a univariable logistic regression fit.

Support Vector Machines [26] are classifiers that divide data instances of different categories with a linear boundary supported by a very clear gap (called maximum margin). They can be optimised via different internal algorithms, therefore a parameter search is often recommended. Support vector machines can efficiently perform a non-linear classification using a so-called "kernel trick" which maps their inputs into feature spaces of higher dimensions. This solution however is more difficult to interpret. In this study we adopted a linear kernel and the nu-classification, optimizing the parameter $n u$ in the value range [0.02, 0.4], with a step size of 0.01 for values below 0.1 , and a step of 0.05 for values above 0.1, using e1071 library [27] in the R software.

Decision trees are non-linear graphical models that take the form of a flow chart. They are a "white-box" method because they produce multiple decision pathways in a tree form that can be easily interpretable [28]. Decision trees consist of nodes which represent input variables, and edges branching from the nodes dependent on possible values of those input variables. Each terminal node (leaf) represents the value of the target variable given the values of the input variables after following the path from the root to the leaf. A decision tree is usually grown by starting from the whole population, looking at the most discriminative variable to predict a desired outcome (which becomes a node), and splitting the data based on a cut-off value of this variable (inducing an edge). In our analysis, we adopted the party package of decision tree learning [29] within the R software.

A single decision tree often does not yield satisfactory prediction performance. To improve performance, multiple different trees can be aggregated, and this takes the general name of a tree ensemble. A weel-recognised tree ensemble method is the random forest [30], which infers different decision trees via resampling and randomization, producing an average prediction from all trees. We used the randomForest package of $\mathrm{R}$ [31]. The combination of several trees makes the method more powerful, but also more difficult to interpret than a single decision tree. Another ensemble method is the AdaBoost [32], which fits several "weak" learners, such as decision trees with only a small number of pathways, and weights them based on performance on data subsets. We adopted the RWeka AdaBoost version of $\mathrm{R}$ [25].

We performed the complete cases analysis with all methods and used three different approaches for imputation of missing values: i) addition of a categorical variable encoding the presence of a missing value; ii) substitution with the overall population mode for binary attributes and mean for numeric ones; iii) non-linear imputation based on random forests [33].

Models' performance was analysed by means of sensitivity (true positive rate), specificity (true negative rate), and using the area under the receiver operating characteristic (AUC), which is a combined indicator of sensitivity and specificity, equal to the probability that a classifier will rank a randomly chosen positive instance higher than a randomly chosen negative one [34]. The robustness of performance was assessed via bootstrapping [35], a validation technique based on random data resampling with replacement (here, 50 times); we used the very conservative outof-bag estimator which calculates errors on unseen data. To assess the entity of the difference between means of two performance distributions, a modified $t$-test was used, penalising the degrees of freedom due to sample overlap [36]. Variable importance analysis for the different machine learning techniques was carried out as follows: odds ratio/AIC with associated $\mathrm{p}$-values for both multivariable and univariable logistic regression; conditional independence split rule + pruning for decision trees as implemented in ctree function of party library in R [29]; Gini index for random forest; decrease in AUC by single feature elimination for AdaBoost and support vector machines.

\section{Results}

\section{Study population}

Table 1 the characteristics of the study population (487 patients, 912 eyes). The percentage of males was $49.5 \%$. The mean (std. dev.) age was 65.3 (14.9) years in males, and 70.5 (12.7) years in females. The proportion of AMD diagnoses was $22.5 \%$ in males and $31.4 \%$ in females $(\mathrm{p}<0.0001$ by a test for equality of proportions). Among AMDdiagnosed patients, dry and wet AMD had a prevalence of 9.7\% and $38.1 \%$ respectively. Healthy subjects accounted for $31.6 \%$ of the study population. 
Table 1 Population's characteristics

\begin{tabular}{|c|c|c|c|c|}
\hline Parameter & M & $\mathbf{F}$ & Total & Missing \\
\hline Number of patients (\%) & $241(49.5 \%)$ & $246(50.5 \%)$ & 487 & / \\
\hline Number of eyes (\%) & $444(48.7 \%)$ & $468(51.3 \%)$ & 912 & / \\
\hline Number of healthy eyes (\%) & $138(31.1 \%)$ & $150(32.1 \%)$ & $288(31.6 \%)$ & / \\
\hline Age (mean+/-std) & $65.3+/-14.9$ & $70.5+/-12.7$ & $68+/-14.1$ & / \\
\hline Soft drusen positive (\%) & $21(6.4 \%)$ & $62(17.9 \%)$ & $83(12.4 \%)$ & $240(26.3 \%)$ \\
\hline Macular scar positive (\%) & $19(5.8 \%)$ & $32(9.2 \%)$ & $51(7.6 \%)$ & $237(26 \%)$ \\
\hline RPE defect/pigment mottling positive (\%) & $82(25.2 \%)$ & $118(34.1 \%)$ & $200(29.8 \%)$ & $240(26.3 \%)$ \\
\hline Depigmentation area positive (\%) & $95(29.1 \%)$ & $134(38.7 \%)$ & $229(34.1 \%)$ & $240(26.3 \%)$ \\
\hline Subretinal fluid positive (\%) & $79(21.8 \%)$ & $50(13.4 \%)$ & $129(17.5 \%)$ & $176(19.3 \%)$ \\
\hline Macular tickness (mean+/-std) & $297.4+/-64.8$ & $277+/-54.5$ & $286.8+/-60.5$ & $149(16.3 \%)$ \\
\hline Subretinal fibrosis positive (\%) & $18(5.7 \%)$ & $26(7.5 \%)$ & $44(6.6 \%)$ & $248(27.2 \%)$ \\
\hline Subretinal hemorrhage positive (\%) & $16(5.2 \%)$ & 19 (5.9\%) & $35(5.5 \%)$ & $281(30.8 \%)$ \\
\hline AMD diagnosis (\%) & $100(22.5 \%)$ & $147(31.4 \%)$ & $247(27.1 \%)$ & / \\
\hline
\end{tabular}

Percentages of attributes are calculated considering the total of eyes with no missing values for the specific attribute in the strata (Male/Female) and total.

Table 2 shows the prevalence of non-AMD and AMD subjects, stratified by soft drusen variable, Depigmentation area and RPE defect/pigment mottling, which are the most discriminant variables for AMD. Contingency tables are available in the Additional files 1 and 2 for all the other variables.

\section{Performance of statistical learning methods}

Table 3 shows the predictive performance of models trained on the dataset (912 eyes from 487 patients) upon the bootstrap validation. Results were computed using complete cases and the three different imputation techniques described in the Methods section. Performance is shown in terms of AUC, sensitivity and specificity. In regards to AUC, random forest and logistic regression were ranked as the best, followed by AdaBoost, support vector machine, decision tree and one-rule. When considering sensitivity (the percentage of patients who are correctly identified as having AMD), support vector machine was superior, whilst random forest displayed the highest specificity (the percentage of healthy people who are correctly identified as not having AMD).

We executed a formal $t$-test to compare shifts in the average AUCs of methods -specifically, logistic regression, support vector machine, AdaBoost and decision trees against random forest- given the current data, there was

Table 2 Prevalence of diagnoses of retinal diseases in the whole population stratified by soft drusen, depigmentation area and RPE defect/pigment mottling (counting one eye as a single case)

\begin{tabular}{lcccc}
\hline Disease (or healthy status) & $\mathbf{N}(\%)$ & Soft drusen positive N (\%) & Depigmentation area positive N (\%) & $\begin{array}{c}\text { RPE defect/pigment } \\
\text { mottling positive N (\%) }\end{array}$ \\
\hline AMD & $247(27.1 \%)$ & $76(30.8 \%)$ & $136(55.1 \%)$ & $125(50.6 \%)$ \\
Angioid streaks & $5(0.5 \%)$ & $0(0 \%)$ & $3(60 \%)$ & $1(20 \%)$ \\
Central serous chorioretinopathy & $69(7.6 \%)$ & $0(0 \%)$ & $21(30.4 \%)$ & $17(24.6 \%)$ \\
Choroidal hemangioma & $3(0.3 \%)$ & $0(0 \%)$ & $0(0 \%)$ & $0(0 \%)$ \\
Diabetic retinopathy & $126(13.8 \%)$ & $1(0.8 \%)$ & $18(14.3 \%)$ & $11(8.7 \%)$ \\
Distrophy & $24(2.6 \%)$ & $3(12.5 \%)$ & $10(41.7 \%)$ & $9(37.5 \%)$ \\
Epiretinal membrane & $30(3.3 \%)$ & $0(0 \%)$ & $4(13.3 \%)$ & $4(13.3 \%)$ \\
Inflammatory cystoid macular edema & $4(0.4 \%)$ & $0(0 \%)$ & $0(0 \%)$ & $0(0 \%)$ \\
Macroaneurisma & $1(0.1 \%)$ & $0(0 \%)$ & $0(0 \%)$ & $0(0 \%)$ \\
Pathologic myopia & $66(7.2 \%)$ & $0(0 \%)$ & $22(33.3 \%)$ & $12(18.2 \%)$ \\
Retinal artery occlusion & $3(0.3 \%)$ & $0(0 \%)$ & $1(33.3 \%)$ & $1(33.3 \%)$ \\
Retinal vein occlusion & $41(4.5 \%)$ & $0(0 \%)$ & $1(2.4 \%)$ & $2(4.9 \%)$ \\
Uveitis & $5(0.5 \%)$ & $0(0 \%)$ & $1(20 \%)$ & $0(0 \%)$ \\
\hline
\end{tabular}


Table 3 Performance of the machine learning methods in terms of average (+/- std. dev.) sensitivity, specificity and area under the receiver operating characteristic (AUC), applied to the dataset with different missing value imputation techniques (complete cases, categorical variable encoding the missingness, mean/mode imputation, and random forest imputation)

\begin{tabular}{|c|c|c|c|c|c|c|c|}
\hline $\begin{array}{l}\text { Type of imputation on } \\
\text { the dataset }(\mathrm{N}=444)\end{array}$ & $\begin{array}{l}\text { Performance } \\
\text { function }\end{array}$ & One-rule & Decision tree & $\begin{array}{l}\text { Logistic } \\
\text { regression }\end{array}$ & $\begin{array}{l}\text { Random } \\
\text { forest }\end{array}$ & AdaBoost & $\begin{array}{l}\text { Support vector } \\
\text { machine }\end{array}$ \\
\hline \multirow[t]{3}{*}{ Complete cases } & $A \cup C$ & $0.74+/-0.05$ & $0.90+/-0.03$ & $0.93+/-0.04$ & $0.94+/-0.01$ & $0.92+/-0.02$ & $0.92+/-0.03$ \\
\hline & Sensitivity & $0.87+/-0.10$ & $0.88+/-0.07$ & $0.92+/-0.03$ & $0.90+/-0.03$ & $0.91+/-0.02$ & $0.94+/-0.03$ \\
\hline & Specificity & $0.60+/-0.18$ & $0.74+/-0.15$ & $0.70+/-0.08$ & $0.78+/-0.07$ & $0.71+/-0.06$ & $0.67+/-0.07$ \\
\hline \multirow{3}{*}{$\begin{array}{l}\text { Categorical variable } \\
\text { encoding the missingness }\end{array}$} & AUC & $0.73+/-0.04$ & $0.88+/-0.02$ & $0.91+/-0.01$ & $0.92+/-0.02$ & $0.90+/-0.01$ & $0.89+/-0.03$ \\
\hline & Sensitivity & $0.92+/-0.07$ & $0.88+/-0.07$ & $0.92+/-0.03$ & $0.91+/-0.02$ & $0.91+/-0.03$ & $0.93+/-0.03$ \\
\hline & Specificity & $0.42+/-0.05$ & $0.61+/-0.18$ & $0.60+/-0.07$ & $0.68+/-0.06$ & $0.60+/-0.06$ & $0.51+/-0.07$ \\
\hline \multirow[t]{3}{*}{ Mean/mode } & AUC & $0.69+/-0.05$ & $0.85+/-0.02$ & $0.88+/-0.02$ & $0.87+/-0.02$ & $0.87+/-0.02$ & $0.86+/-0.04$ \\
\hline & Sensitivity & $0.94+/-0.05$ & $0.92+/-0.04$ & $0.94+/-0.02$ & $0.93+/-0.02$ & $0.93+/-0.02$ & $0.96+/-0.02$ \\
\hline & Specificity & $0.31+/-0.12$ & $0.56+/-0.10$ & $0.54+/-0.05$ & $0.56+/-0.05$ & $0.53+/-0.05$ & $0.47+/-0.06$ \\
\hline \multirow[t]{3}{*}{ Random forest } & AUC & $0.79+/-0.02$ & $0.95+/-0.02$ & $0.96+/-0.01$ & $0.96+/-0.01$ & $0.96+/-0.01$ & $0.94+/-0.03$ \\
\hline & Sensitivity & $0.97+/-0.04$ & $0.94+/-0.04$ & $0.96+/-0.02$ & $0.94+/-0.02$ & $0.95+/-0.01$ & $0.96+/-0.01$ \\
\hline & Specificity & $0.60+/-0.06$ & $0.78+/-0.09$ & $0.75+/-0.05$ & $0.81+/-0.04$ & $0.76+/-0.04$ & $0.75+/-0.05$ \\
\hline
\end{tabular}

Results are calculated on 50 bootstrap tests, using out-of-bag predictions (in bold the best performance for each characteristic).

not enough evidence against the hypothesis of no difference in mean (whichever imputation method was used, all p-values were $>0.3)$. Instead, the one-rule method had a lower mean AUC than all other methods ( $\mathrm{p}<0.0001$ in all imputation scenarios).

Figure 1 shows receiver operating characteristic curves for each method obtained by averaging the results from the 50 bootstrap tests. As reported in Table 3, random forest and logistic regression curves dominate the others.

Since logistic regression was not inferior to random forest in terms of AUC, we report the model fit in Table 4. Soft drusen, as expected, was the most important variable with an odds ratio (positive vs negative) of 19.3 ( $\mathrm{p}<0.0001$ ). Other relevant variables were: subretinal fluid, subretinal hemorrage, subretinal fibrosisRPE defect/pigment mottling, depigmentation area, and age.

As shown by the overall sensitivity, specificity and AUC results, the decision tree assures fair performance and its structure has high interpretability. The tree is shown in Figure 2 (dataset with mean/mode imputaion for missing values). The tree should be traversed from the root node downwards. Split nodes are evaluated according to the value of the variable of interest and the decision pathway to follow is the corresponding attribute value on

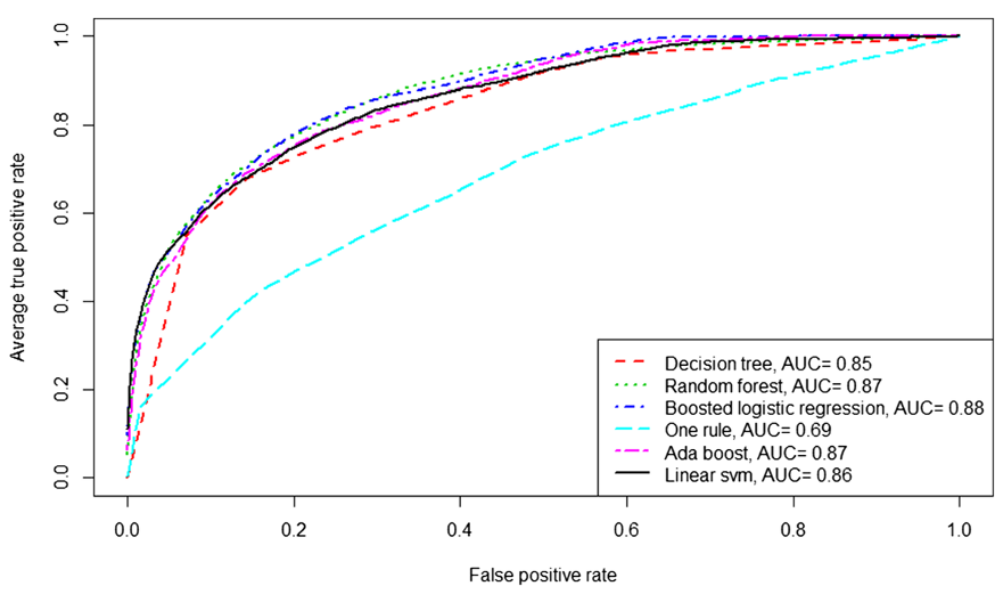

Figure 1 Receiver operating characteristic curves plotting performance of different statistical learning methods, averaging results from 50 bootstrap tests (out-of-bag predictions, dataset imputing mean/mode for missing values). 
Table 4 Odds ratio from fitting the LogitBoost logistic regression on the AMD diagnosis outcome (dataset imputing missing values with population's mean/mode)

\begin{tabular}{|c|c|c|c|c|}
\hline Variable (mode) & Odds ratio & Lower $95 \% \mathrm{Cl}$ & Upper $95 \% \mathrm{Cl}$ & P-value \\
\hline Age (per year older) & 1.09 & 1.07 & 1.11 & $<0.0001$ \\
\hline Gender (M vs F) & 1.05 & 0.71 & 1.57 & 0.7985 \\
\hline Soft drusen (pos vs neg) & 19.30 & 7.82 & 47.65 & $<0.0001$ \\
\hline Macular scar (pos vs neg) & 1.75 & 0.57 & 5.41 & 0.329 \\
\hline RPE defect/pigment mottling (pos vs neg) & 2.20 & 1.20 & 4.04 & 0.0109 \\
\hline Depigmentation area (pos vs neg) & 1.35 & 0.73 & 2.51 & 0.3349 \\
\hline Subretinal fluid (pos vs neg) & 3.21 & 1.70 & 6.08 & 0.0003 \\
\hline Macular tickness (per unit increase) & 1.00 & 0.99 & 1.00 & 0.139 \\
\hline Subretinal fibrosis (pos vs neg) & 4.60 & 1.39 & 15.24 & 0.01245 \\
\hline Subretinal hemorrhage (pos vs neg) & 5.91 & 1.49 & 23.42 & 0.01138 \\
\hline
\end{tabular}

Statistically significant $\mathrm{p}$-values are reported in bold.

the branch. Again, soft drusen had the highest discriminative power (76 eyes out of 83 with a positive soft drusen are diagnosed with AMD) and was selected as the root node. Following soft drusen, the other variables selected as node splits were: age, depigmentation area, subretinal fibrosis, subretinal fluid and RPE defect/pigment mottling,. Notably, the leaf nodes numbered \#2, \#6, 13 (corresponding to the first, third, and seventh bottom terminal nodes from the left) clearly identify sub-groups where the AMD diagnosis is straightforward ( $>80 \%$ with/without AMD), whilst the other leaf nodes represent sub-groups where the AMD diagnosis is present in the range of $20 \%$ to $60 \%$ (nodes \#2, \#9, \#11, \#12), thus not allowing a definitive classification.

Table 5 shows variable importance ranking (mean/ mode imputation) for one rule, random forest, AdaBoost and support vector machine. In agreement with logistic regression and decision tree, soft drusen and age are

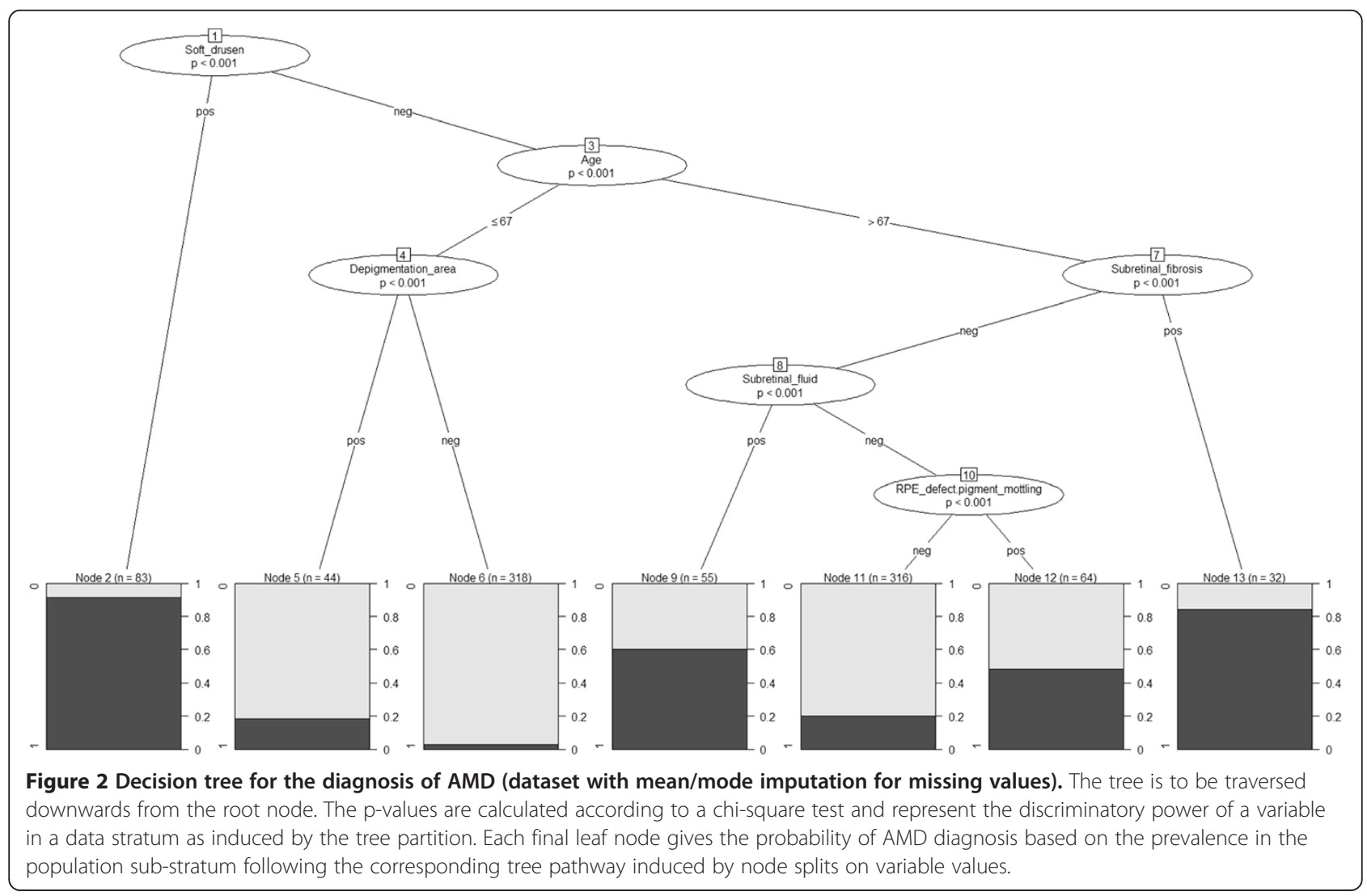




\begin{tabular}{|c|c|c|c|c|}
\hline Ranking & One rule & Random forest & AdaBoost & Support vector machine \\
\hline 1 & Soft drusen & Age & Age & Age \\
\hline 2 & Age & Soft drusen & Soft drusen & Gender \\
\hline 3 & Depigmentation area & Macular tickness & Subretinal fluid & Soft drusen \\
\hline 4 & RPE defect.pigment mottling & Depigmentation area & Subretinal hemorrhage & Subretinal hemorrhage \\
\hline 5 & Subretinal hemorrhage & RPE defect.pigment mottling & RPE defect.pigment mottling & Subretinal fluid \\
\hline 6 & Subretinal fibrosis & Subretinal fibrosis & Gender & Subretinal fibrosis \\
\hline 7 & Macular.scar & Subretinal hemorrhage & Subretinal fibrosis & RPE defect.pigment mottling \\
\hline 8 & Subretinal fluid & Subretinal fluid & Macular.scar & Macular.scar \\
\hline 9 & Gender & Macular.scar & Macular tickness & Macular tickness \\
\hline 10 & Macular tickness & Gender & Depigmentation area & Depigmentation area \\
\hline
\end{tabular}

consistently at the top of the ranking. This is confirmed in all analyses performed with different imputation methods (see Additional files 1 and 2).

\section{Discussion}

This work investigated several machine learning approaches for deriving an automated system for AMD diagnosis, using clinical attributes identified by a medical retinal specialist during a routine visit. The study population was monitored via an Electronic Health Record employed by a single clinical practice in Genoa, Italy.

We compared "white-box" (i.e. more interpretable) vs. "black-box" (i.e. less interpretable) techniques in terms of predictive performance. We found that higher complexityhigher performance does not necessarily hold in all contexts and a performance-complexity compromise may be found. For example, the simplest one-rule model yielded an average of $74 \%$ AUC in experiments, whilst the more complex, fully non-linear random forest and AdaBoost yielded 92\% AUC. Whilst a single variable cannot be used for reliable diagnosis, logistic regression (average AUC of 92\%) and decision tree (90\% average AUC) were not inferior to random forest. These two modelling techniques combined interpretability and performance, as shown through the odds ratio table and tree diagram, which can be easily followed by a clinician during the diagnostic process.

Physicians must be involved in the decision about what type of system will be used in practice because without their agreement and trust, such a system risks not to being used. Generally, from the perspective of a fully automated system, where a computer program performs all calculations, the main driver should not be the interpretability of the model, but the overall performance. For example, if a black-box ensures an increment $10 \%$ over a white-box method and if this $10 \%$ is clinically relevant (if properly validated in a prospective trial), then the choice should be obvious. But in the case where model performances are comparable, such as the results reported in this study referring, the white-box is a preferable alternative.

The methods proved to be robust to handle missing values and obtained performances did not change significantly varying the imputation methods. Although complete cases and random forest imputation yielded better performance than the other methods, we think that the most reliable analysis is the one with mean/ mode imputation. In fact, the clinicians that performed the analysis suggested that the majority of missing values are likely to be clinical signs that they did not identify during encounters, and thus negative values were not recorded in the system to save time, starting from the assumption that if a sign had been identified a positive value would have been registered in the system.

This study has some limitations. The study population itself is not large (487 subjects and 912 eyes) and includes only patients from a local regional area. Although the out of bag error estimator is very conservative, a way in which the generalisation error could be challenged is by considering the study population and the diagnostic process as regionally biased: for instance, by assuming that the population of Genoa and neighbouring areas (Liguria) is different from Italy (or worldwide) and that doctors make diagnoses differently. Accordingly, it would be interesting to see how the automated diagnostic algorithms would behave on patients from other countries. This would unveil indirectly the differences in the population characteristics and in the gold-standard diagnostic procedures. Performance would be affected only by using two different systems trained on two different populations, whilst one could infer a new integrated model which takes into account such regional differences and aims at the same diagnostic ability in different settings.

A more thorough analysis of missing values could be performed in order to identify the characteristic of missingness 
and their relevancy. Also, further investigation on intrapatient correlation is warranted. Using two records from the same patient (i.e. both eyes) may yield to correlated observations, thus overall performance results may be affected by this correlation (higher than in reality). We carried out a series of additional experiments, not shown in this paper, using only single-patient and single-eye data (out of 912 eyes, we selected 487 eyes pertaining to 487 different patients randomly, for 10 times). The analysis on this uncorrelated data was consistent with the main results in terms of sensitivity but yielded slightly lower specificity. This is most likely due to the smaller sample size (487 vs. 912 ) rather than the effect of correlation in the main dataset however a larger study population is required to verify this claim. A larger population and attribute set may also help to refine the model and allow prediction of different subtypes of AMD, for instance neurovascular AMD.

\section{Conclusions}

From a rationale point of view, the utility of the system -for now- is to determine which are the diagnostic processes followed by the physicians, since the data were crosssectional and the diagnoses were made by doctors during visits. We found that even by using powerful nonlinear machine learning models, we could not exactly all the consistent sets of diagnostic pathways. Therefore, even in presence of standardised guidelines, physicians may follow different diagnostic routes (as those shown in the decision tree) which in some cases lead to ambiguities (see the proportions of patients with/without AMD in the tree leaves which correspond to specific variable strata). When longitudinal data and new background variables (e.g. other image processed data) will be available, an automated system will help not only in identifying such different decision paths (with an augmented information set), but also in making early diagnoses feasible and better differentiating those ambiguous subsets of patients.

In fact, we are in an era where diagnosis of AMD is most commonly pursued by image analysis, yet digital image processing techniques embedded in commercial OCT systems are still in their infancy. Imaging can be integrated with information coming from data collected during everyday clinical practice by medical retina specialists. From a technological perspective, implementing such a diagnostic model into a computer program would not be a hurdle, and using a multi-platform language (e.g. Java or Python) could facilitate the integration into Electronic Health Record systems coming from different vendors. If the model were also one of those white-box (logistic regression or decision tree in this case), a graphical user interface showing the diagnostic pathway or variable importance could be provided. Such a program could be used in real-time by physicians as a support to diagnosis as well as for educational purposes.

\section{Additional files}

Additional file 1: Cross tabulation between AMD and all covariates.

Additional file 2: Variable importance derived for each missing value imputation technique.

\section{Competing interests}

The authors declare that they have no competing interests.

\section{Authors' contributions}

PF, MP carried out the experiments, revised the results, drafted and revised the manuscript. DOS revised the manuscript giving relevant intellectual contribution and gave final approval for submission. MN designed the experiment, revised results and manuscript. $C T, P W, M G$ revised the manuscript giving relevant intellectual contribution. MB developed and managed the web platform to collect the data and extracted information from the database. All authors read and approved the final manuscript.

\section{Acknowledgements}

This study was supported in part by the DINOGMI and DIBRIS Fund of the University of Genova, Italy.

\section{Author details}

${ }^{1}$ Centre for Health Informatics, City University London, London, UK. ${ }^{2}$ Centre for Health Informatics, University of Manchester, Manchester, UK. ${ }^{3}$ DIBRIS, University of Genoa, Genoa, Italy. ${ }^{4}$ Di.N.O.G.Mi, University of Genoa, L.go P. Daneo 3, Genoa 16132, Italy. ${ }^{5}$ CEBR, University of Genoa, Genoa, Italy. ${ }^{6}$ NIHR Primary Care Patient Safety Translational Research Centre, University of Manchester, Manchester, UK. ${ }^{7}$ Health eResearch Centre, University of Manchester, Manchester, UK.

Received: 6 May 2014 Accepted: 3 January 2015 Published: 27 January 2015

\section{References}

1. Bressler NM. Age-Related Macular Degeneration Is the Leading Cause of Blindness. J Am Med Assoc. 2004;291:1900.

2. Rein DB, Wittenborn JS, Zhang X, Honeycutt AA, Lesesne SB, Saaddine J. Forecasting age-related macular degeneration through the year 2050: the potential impact of new treatments. Arch Ophthalmol. 2009;127:533-40.

3. Lamoureux EL, Mitchell P, Rees G, Cheung G, Yeo I, Lee SY, et al. Impact of early and late age-related macular degeneration on vision-specific functioning. Br J Ophthalmol. 2011;95:666-70.

4. Hee MR, Baumal CR, Puliafito CA, Duker JS, Reichel E, Wilkins JR, et al. Optical coherence tomography of age-related macular degeneration and choroidal neovascularization. Ophthalmol. 1996;103:1260-70.

5. Kanagasingam Y, Bhuiyan A, Abràmoff MD, Smith RT, Goldschmidt L, Wong TY. Progress on retinal image analysis for age related macular degeneration. Prog Retin Eye Res. 2014;38:20-42.

6. Hee MR, Puliafito CA, Duker JS, Reichel E, Coker JG, Wilkins JR, et al. Topography of diabetic macular edema with optical coherence tomography. Ophthalmol. 1998;105:360-70.

7. Pifer EA, Sittig DF. Improving Outcomes with Clinical Decision Support: An Implementer' s Guide. Soc: In Chicago Healthc. Inf. Manag. Syst; 2005.

8. Quellec G, Russell SR, Scheetz TE, Stone EM, Abràmoff MD. Computational quantification of complex fundus phenotypes in age-related macular degeneration and Stargardt disease. Invest Ophthalmol Vis Sci. 2011;52:2976-81.

9. Sivasankari S, Narasimhan K, Ramya S. Automated diagnosis of ARMD. Int J Eng Technol. 2013;5:1462-4.

10. Schlanitz FG, Baumann B, Spalek T, Schütze C, Ahlers C, Pircher M, et al. Performance of automated drusen detection by polarization-sensitive optical coherence tomography. Investig Ophthalmol Vis Sci. 2011;52:4571-9.

11. Pauleikhoff D, Barondes MJ, Minassian D, Chisholm IH, Bird AC. Drusen as risk factors in age-related macular disease. Am J Ophthalmol. 1990;109:38-43.

12. Ferris FL, Wilkinson CP, Bird A, Chakravarthy U, Chew E, Csaky K, et al. Clinical classification of age-related macular degeneration. Ophthalmol. 2013;120:844-51

13. Mehryar M, Afshin R, Ameet T. Foundations of Machine Learning. Cambridge, Massachussets, London, England: The MIT Press; 2012. 
14. Zheng Y, Hijazi MHA, Coenen F. Automated "disease/no disease" grading of age-related macular degeneration by an image mining approach. Invest Ophthalmol Vis Sci. 2012;53:8310-8.

15. Serrano-Aguilar P, Abreu R, Antón-Canalís L, Guerra-Artal C, Ramallo-Fariña Y, Gómez-Ulla F, et al. Development and validation of a computer-aided diagnostic tool to screen for age-related macular degeneration by optical coherence tomography. Br J Ophthalmol. 2012;96:503-7.

16. Hijazi MHA, Coenen F, Zheng Y. Data mining techniques for the screening of age-related macular degeneration. Knowl Base Syst. 2012;29:83-92.

17. Liu YY, Ishikawa H, Chen M, Wollstein G, Duker JS, Fujimoto JG, et al. Computerized macular pathology diagnosis in spectral domain optical coherence tomography scans based on multiscale texture and shape features. Investig Ophthalmol Vis Sci. 2011;52:8316-22.

18. Kankanahalli S, Burlina PM, Wolfson Y, Freund DE, Bressler NM. Automated classification of severity of age-related macular degeneration from fundus photographs. Invest Ophthalmol Vis Sci. 2013;54:1789-96.

19. Bonetto M, Fraccaro P, Nicolo M, Giacomini M. A web-tool to manage ophthalmologic electronic health records. In: IEEE-EMBS Int. Conf. Biomed. Heal. Informatics. 2014. IEEE; 2014:346-349.

20. Fraccaro P, Dentone C, Fenoglio D, Giacomini M. Multicentre clinical trials' data management: a hybrid solution to exploit the strengths of electronic data capture and electronic health records systems. Inform Health Soc Care. 2013;38:313-29.

21. Murdoch IE, Morris SS, Cousens SN. People and eyes: statistical approaches in ophthalmology. Br J Ophthalmol. 1998;82:971-3.

22. Bishop CM. Pattern Recognition and Machine Learning. Berlin, Heidelberg: Springer; 2006. p. 738.

23. Hosmer Jr DW, Lemeshow S. Applied Logistic Regression. Hoboken New Jersey: John Wiley \& Sons; 2004. p. 392.

24. Friedman J, Hastie T, Tibshirani R. Additive logistic regression: a statistical view of boosting (With discussion and a rejoinder by the authors). Ann Stat. 2000;28:337-407.

25. Hornik K, Buchta C, Zeileis A. Open-source machine learning: R meets Weka. Comput Stat. 2008;24:225-32.

26. Cortes C, Vapnik V. Support-vector networks. Mach Learn. 1995;20:273-97.

27. Dimitriadou E, Hornik K, Leisch F, Meyer D, Weingessel A. SVM, e1071 Package, The R project for statistical computing. 2004.

28. Breiman L, Friedman JH, Olshen RA, Stone CJ. Classification and regression trees. Advanced B. Monterey: CA; 1984.

29. Hothorn T, Hornik K, Zeileis A. party: A Laboratory for Recursive Partytioning. http://cran.r-project.org/web/packages/party/index.htm

30. Breiman L. Random Forests. Mach Learn. 2001;45:5-32.

31. Liaw A, Wiener M. Classification and Regression by randomForest. R News. 2002;2:18-22.

32. Freund Y, Schapire RE. A Decision-Theoretic Generalization of On-Line Learning and an Application to Boosting. J Comput Syst Sci. 1997;55:119-39.

33. Stekhoven DJ, Bühlmann P. MissForest-non-parametric missing value imputation for mixed-type data. Bioinformatics. 2012;28:112-8.

34. Fawcett T. An introduction to ROC analysis. Pattern Recognit Lett. 2006;27:861-74.

35. Breiman L. Bagging predictors. Mach Learn. 1996;24:123-40.

36. Nadeau C, Bengio Y. Inference for the Generalization Error. Mach Learn. 2003;52:239-81.

\section{doi:10.1186/1471-2415-15-10}

Cite this article as: Fraccaro et al: Combining macula clinical signs and patient characteristics for age-related macular degeneration diagnosis: a machine learning approach. BMC Ophthalmology 2015 15:10.

\section{Submit your next manuscript to BioMed Central and take full advantage of:}

- Convenient online submission

- Thorough peer review

- No space constraints or color figure charges

- Immediate publication on acceptance

- Inclusion in PubMed, CAS, Scopus and Google Scholar

- Research which is freely available for redistribution 